\title{
AN INDIVIDUALIZED LOW-INTENSITY WALKING CLINIC LEADS TO IMPROVEMENT IN FRAILTY CHARACTERISTICS IN OLDER VETERANS
}

\author{
S.E. ESPINOZA ${ }^{1,2,3}$, B. ORSAK ${ }^{2,3}$, C.-P. WANG ${ }^{2,4}$, D. MACCARTHY ${ }^{2,3}$, D. KELLOGG ${ }^{1,2,3}$, B. POWERS ${ }^{1,2,3}$, \\ A. CONDE ${ }^{2,3}$, M. MORIS ${ }^{2,3}$, P.R. PADALA ${ }^{5,6,7}, K^{2}$ P. PADALA ${ }^{5,7}$

\begin{abstract}
1. Department of Medicine, Division of Geriatrics, Gerontology \& Palliative Medicine, University of Texas Health Science Center at San Antonio; 2. Sam and Ann Barshop Institute for Longevity and Aging Studies, University of Texas Health Science Center at San Antonio; 3. Geriatric Research, Education \& Clinical Center (GRECC), South Texas Veterans Health Care System; 4. Department of Epidemiology and Biostatistics, University of Texas Health Science Center at San Antonio; 5. GRECC, Central Arkansas Veterans Healthcare System; 6. Department of Psychiatry, University of Arkansas for Medical Sciences; 7. Department of Geriatrics, University of Arkansas for Medical Sciences.
\end{abstract} \\ Corresponding author: Sara Espinoza, MD, 7703 Floyd Curl Drive, Mail Code 7875, San Antonio, TX 78223, Telephone: 210-617-5197, E-mail: espinozas2@uthscsa.edu, \\ FAX: 210-949-3060
}

\begin{abstract}
Background: Sedentary lifestyle leads to worse health outcomes with aging, including frailty. Older adults can benefit from regular physical activity, but exercise promotion in the clinical setting is challenging. Objectives: The objective of this clinical demonstration project was to implement a Geriatric Walking Clinic for older adults and determine whether this clinical program can lead to improvements in characteristics of frailty. Design: This was a clinical demonstration project/quality improvement project. Setting: Outpatient geriatrics clinic at the South Texas Veterans Health Care System (STVHCS). Participants: Older Veterans, aged $\geq 60$ years. Intervention: A 6-week structured walking program, delivered by a registered nurse and geriatrician. Patients received a pedometer and a comprehensive safety evaluation at an initial face-to-face visit. They were subsequently followed with weekly phone calls and participated in a final face-to-face follow-up visit at 6 weeks. Measurements: Grip strength (handheld dynamometer), gait speed (10-ft walk), Timed Up and Go (TUG), and body mass index (BMI) were assessed at baseline and follow-up. Frailty status for gait speed was assessed using Fried criteria. Results: One hundred eighty five patients completed the program (mean age: $68.4 \pm 7$ years, $88 \%$ male). Improvements from baseline to follow-up were observed in average steps/day, gait speed, TUG, and BMI. Improvement in gait speed $(1.13 \pm 0.20$ vs. $1.24 \pm 0.23$ meter/second, $\mathrm{p}<0.0001)$ resulted in reduced odds of meeting frailty criteria for slow gait at follow-up compared to the baseline examination (odds ratio $=0.31$, 95\% confidence interval: $0.13-0.72, \mathrm{p}=0.01)$. Conclusions: Our findings demonstrate that a short duration, low-intensity walking intervention improves gait speed and TUG. This new clinical model may be useful for the promotion of physical activity, and for the prevention or amelioration of frailty characteristics in older adults.
\end{abstract}

Key words: Frailty, physical activity, gait speed.

\section{Introduction}

Over the past two decades, the geriatrics community has recognized frailty as a geriatric syndrome that dramatically affects physical function, quality of life, and leads to increased health care costs. The estimated cost of frailty in the U.S. was over $\$ 18$ billion in 2000 (1). Several studies have shown that frailty leads to falls, hospitalization, disability, and an increased risk of nursing home placement (2-4). Older adults are prone to adopt a sedentary lifestyle with increasing age (5), which can contribute to poor health and disability (6). Low physical activity is one of the key frailty characteristics in the Fried frailty model; others include unintentional weight loss, slow gait, muscle weakness, and self-reported exhaustion $(2,7)$. Given the enormous costs associated with frailty, both personal and economic, a clinical intervention that prevents or delays frailty that is easy to deliver in a clinical setting would have a major impact on our society.

Exercise is known to have major health benefits for older adults, including improvement in frailty-related measures, such as gait speed, and improvement in the ability to perform activities of daily living $(8,9)$. Even the most frail older adults Received September 19, 2018

Accepted for publication March 20, 2019 can have significant improvements in strength and physical function with exercise (10). While the benefits of exercise are known, few clinical trials have focused on the Veteran population, and few studies have examined the feasibility of implementing a physical activity intervention in a clinical setting. We do know, however, that engagement and regular contact with clinical staff and physicians along with an exercise prescription can have a greater impact on future exercise success $(11,12)$.

To address this and to promote an active lifestyle in older Veterans at South Texas Veterans Health Care System (STVHCS), we developed an outpatient intervention to promote physical activity in older Veterans. This intervention was modeled after the Little Rock Geriatric Walking Clinic (GWC) while maintaining a specific focus of the local site which was to improve components of frailty (13). The Little Rock GWC is a patient-centric program that implements a comprehensive approach to engage older Veterans in a long-term program of regular physical activity primarily in the form of walking. This clinic uses proven strategies, such as motivational counseling, follow-up phone calls from a nurse, and self-monitoring using pedometers. Here we report short-term results of this program 


\section{GERIATRICS WALKING CLINIC IMPROVES GAIT SPEED}

from the 6-week follow-up.

\section{Methods}

\section{Patient Population}

The Geriatric Walking Clinic is a 6-week walking program designed to encourage increased physical activity in older adults through regular walking. Patients from the outpatient services at STVHCS were eligible for participation if they were 60 years or older, willing to walk for exercise, and willing to accept weekly phone calls. Patients were recruited via: 1) flyers/brochures placed in patient waiting areas within the main hospital (Audie L. Murphy Memorial VA Hospital) and Community-based Outpatient Clinics (CBOCs) within STVHCS; 2) community events and health fairs for Veterans within STVHCS; 3) an electronic kiosk placed in the Geriatric Evaluation and Management (GEM) Clinic waiting room with electronic flyers; and 4) referrals from primary care providers (PCPs) through a consult within the electronic medical record system (CPRS), which was available to all PCPs at STVHCS. This project was approved as non-research/quality improvement by the Institutional Review Board at the University of Texas Health Science Center at San Antonio, which serves the STVHCS, and consent was not required.

\section{Patient Screening}

Patients were evaluated for safety for walking exercise based on the National Institute of Aging's (NIA) Exercise Assessment and Screening for You (EASY) criteria (14) and additional medical contraindications. The NIA EASY is a six-item screening tool that helps in selecting older adults for safe participation in an exercise program. The tool includes questions regarding chest pain and tightness during physical activity, dizziness, high blood pressure, pain, stiffness and swelling of joints, falls or feeling unsteady while walking, or any other reason the patient would be concerned about starting a physical activity program. Specific medical contraindications were unstable angina, severe left main coronary artery disease, end stage congestive heart failure (ejection fraction $<30 \%$ ), severe aortic valvular disease, uncontrolled cardiac arrhythmia, uncontrolled hypertension (systolic $>180 \mathrm{mmHg}$ or diastolic $>100 \mathrm{mmHg}$ ), large abdominal aortic aneurysm, severe shortness of breath, cognitive impairment that interferes with compliance, stroke within the prior six months, uncontrolled diabetes mellitus (Hemoglobin A1c [HbA1c] >10\%), and pain limiting walking. All subjects were seen and evaluated by a geriatrician who reviewed their medical history and performed an evaluation to determine appropriateness for participation in the program.

\section{Clinical Intervention}

The clinic involved an initial baseline face-to-face visit, weekly telephone follow-up calls during the period of the intervention, and a follow-up 6-week face-to-face visit. At the baseline clinic visit, patients met individually with a registered nurse (RN) (who was also a certified diabetes educator) and a geriatrician to screen for eligibility and safety for regular walking, perform baseline assessments (see Table 1), support the patient in self-setting individualized goals for the program, issue a pedometer, and train the patient on the use of the pedometer. Patients also received individualized counseling on healthy food intake along with educational materials about benefits of walking at the baseline visit.

During the first week of the program, patients were instructed to perform usual activities and not to change their routine, in order to establish a baseline average step count per day. Weekly telephone calls were made by the RN to inquire about daily step counts; troubleshoot barriers to walking; offer suggestions, encouragement, and counseling; and help the patient establish a goal for an increase in daily step counts (targeting a 5-10\% increase each week).

At approximately 6 weeks, depending on patient availability, patients were seen for a face-to-face visit for review of progress and re-assessment of physical measurements. In order to facilitate continued lifestyle change and walking for exercise, patients were encouraged and provided education on Veterans Administration (VA) and community resources available for continued exercise. At the follow-up visit, patients were encouraged to continue to walk and monitor using their pedometers, received an additional phone call one month later, and received a certificate of completion.

\section{Assessments}

Several assessments were measured at either the baseline, follow-up, or both, as shown in Table 1. At baseline, components of frailty measured included unintentional weight loss, usual levels of physical activity (15), self-reported exhaustion, Timed Get Up and Go (TUG), gait speed, and grip strength. The Fried frailty phenotype criteria (3) were used to determine whether patients met standardized criteria for the physical characteristics of frailty, including weakness (grip strength) and slowness (10-foot walk). The cut-points used were standardized in the San Antonio Longitudinal Study of Aging, which is ethnically similar to our clinic population (7) and are provided in Appendix Table 1. The TUG and gait speed measured lower extremity strength at baseline and follow-up (16). Follow-up measurements of exhaustion and physical activity were not conducted. Therefore, change in meeting frailty classification could not be assessed for exhaustion or physical activity. Although weight loss with the intervention was assessed, unintentional weight loss per se would not be expected to change within the time frame of the intervention. Therefore, formal assessment of change in frailty criteria from baseline to follow-up was conducted for gait speed and grip strength only. A brief evaluation of cognitive function was assessed at the baseline clinic visit using either the CLOX (17) or the Mini Cog (18) assessments to exclude participants with severe cognitive impairment that may hinder their participation. 


\section{THE JOURNAL OF FRAILTY \& AGING}

Table 1

Baseline and follow-up assessments in the Geriatrics Walking Clinic

\begin{tabular}{lll}
\hline Assessment & Baseline & Follow-up \\
\hline Blood pressure, height, weight & $\mathrm{X}$ & $\mathrm{X}$ \\
Cognitive assessment using either CLOX or Mini Cog ${ }^{\mathrm{a}}$ & $\mathrm{X}$ & \\
Unintentional weight loss, physical activity, fatigue $^{\mathrm{b}}$ & $\mathrm{X}$ & \\
Gait Speed, grip strength, timed up and go test $^{\text {X }}$ & $\mathrm{X}$ & $\mathrm{X}$ \\
\hline
\end{tabular}

a. To exclude patients with significant cognitive impairment from participating in the clinic; $b$. To establish the diagnosis of frailty at baseline

\section{Statistical Analysis}

Summary statistics were used to characterize the patient population. Change in steps taken per day, gait speed, TUG, BMI, and grip strength from baseline to follow-up at 6 weeks was examined using paired t-tests. To account for correlation of repeated measures, the generalized estimating equation (GEE) technique assuming a binomial distribution with the logit link was used to determine the changes in frailty classification (not frail vs. frail) in slowness and weakness based on walking speed and grip strength from baseline to the end of the program. Both the unadjusted GEE analysis (or asymptotic unconditional McNemar test)[19] and the adjusted results are reported. The covariates included in the adjusted model are age, education, diabetes, and baseline physical activity.

\section{Results}

Patient characteristics are shown in Table 2. Mean age ( \pm SD) was $68.4 \pm 6.8$ years, $88 \%$ were male, and the majority were Hispanic $(42.7 \%)$. The majority of the patients were obese $(61.8 \%)$ and had diabetes $(55.7 \%)$. Follow-up information is provided only for those who completed the follow-up assessments $(\mathrm{N}=157) ; 28$ individuals examined at baseline did not complete the follow-up exam (15\%). There were no major side effects or injuries that occurred as a result of the intervention. As shown in Table 3, from baseline to followup we observed a significant increase in the number of steps taken per day and significant improvements in gait speed (improved by $0.096 \pm 0.017$ meter/second), TUG (improved by 0.8 second), and BMI (improved by $0.3 \mathrm{~kg} / \mathrm{m} 2$ ). The average number of steps per day after the intervention increased by $1,426$ ( $\mathrm{p}<0.001)$ steps in the unadjusted analysis, and by 1,523 steps $(\mathrm{p}<0.001)$ in the adjusted analysis. No significant change was observed in grip strength from baseline to followup. Thirteen patients met the frailty criterion of low gait speed at baseline. Nearly $85 \%(\mathrm{~N}=11)$ of them improved their gait speed sufficiently so as to not meet the frailty criteria at the 6 -week follow-up visit. Improvement in gait speed $(1.13 \pm 0.20$ vs. $1.24 \pm 0.23$ meters/second, $p<0.0001)$ resulted in reduced number of patients meeting criteria for frailty at the follow-up (10.3\% met criteria at baseline vs. $3.5 \%$ met criteria following the intervention). This resulted in reduced odds of meeting frailty criteria for slow gait at follow-up compared to the baseline examination with an unadjusted odds ratio (OR) of $0.31,95 \%$ confidence interval $(\mathrm{CI}): 0.13-0.72, \mathrm{p}=0.0062$. After adjustment for covariates, the adjusted OR was $0.26,95 \% \mathrm{CI}$ : $0.10-0.69, \mathrm{p}=0.0063$. This suggests a $74 \%$ reduced odds of meeting frailty criteria for gait speed. There was limited, non-significant improvement in grip strength at the followup compared to the baseline $(31.7 \pm 9.8 \mathrm{~kg}$ vs. $31.2 \pm 9.9 \mathrm{~kg}$, p $=0.1765)$. This resulted in $37.2 \%$ meeting frailty criteria for grip strength post-intervention compared to $38.5 \%$ meeting frailty criteria at baseline. The odds of meeting frailty criteria for grip strength at follow-up compared to the baseline examination was not significant in either the unadjusted model $(\mathrm{OR}=0.83, \mathrm{p}$ $=0.147)$ or the adjusted model $(\mathrm{OR}=0.81, \mathrm{p}=0.149)$.

Table 2

Patient characteristics $(\mathrm{N}=185)$

\begin{tabular}{|c|c|}
\hline & Mean (SD) or $n(\%)$ \\
\hline Age, years (range: $52-89$ ) & $68.4(6.8)$ \\
\hline Male sex & $163(88.0)$ \\
\hline \multirow{3}{*}{$\begin{array}{ll}\text { Ethnic group: } & \text { Hispanic } \\
& \text { Non-Hispanic White } \\
& \text { African American }\end{array}$} & $79(42.7)$ \\
\hline & $75(40.5)$ \\
\hline & $27(14.6)$ \\
\hline Education, years, (range: 5-20) & $13.8(2.5)$ \\
\hline Body mass index (BMI), $\mathrm{kg} / \mathrm{m} 2$, (range: $19.9-52.9 \mathrm{~kg} / \mathrm{m}^{2}$ ) & $32.2(5.6)$ \\
\hline \multicolumn{2}{|l|}{ Body mass index category } \\
\hline Normal (BMI $18.5-24.9 \mathrm{~kg} / \mathrm{m} 2)$ & $11(5.9)$ \\
\hline Overweight (BMI $25-29.9 \mathrm{~kg} / \mathrm{m} 2)$ & $60(32.3)$ \\
\hline Obese $(\mathrm{BMI} \geq 30 \mathrm{~kg} / \mathrm{m} 2)$ & $115(61.8)$ \\
\hline Tobacco use, current & $12(6.5)$ \\
\hline Hypertension & $150(81.1)$ \\
\hline Diabetes & $103(55.7)$ \\
\hline Coronary artery disease & $40(21.6)$ \\
\hline Stroke & $6(3.2)$ \\
\hline Congestive heart failure & $11(5.9)$ \\
\hline Fallen in the last year & $64(34.6)$ \\
\hline Physical activity, kcal/week, (range: 0-16124.2) & $4605.0(3317.5)$ \\
\hline Timed up and go, seconds, (range: $6.7-19.5$ ) & $10.5(2.4)$ \\
\hline Walking speed (10 foot walk), meters/second, (range: $0.65-1.69$ ) & $1.13(0.20)$ \\
\hline Grip strength, kg, (range: 9.4-59.3) & $31.6(9.6)$ \\
\hline
\end{tabular}

\section{Discussion}

Our results demonstrate the feasibility of a low-impact activity promotion clinic. We also demonstrate that a clinic that promotes walking as physical activity in older adults leads to improvements in gait speed, TUG, and BMI even at a short follow-up of six weeks. The improvement in gait was associated with reduction in the proportion of individuals who met gait speed criteria for frailty from baseline to follow-up. 


\section{GERIATRICS WALKING CLINIC IMPROVES GAIT SPEED}

\section{Table 3}

Change in characteristics from baseline to follow-up at 6 weeks $(\mathrm{N}=157)$

\begin{tabular}{lcccc}
\hline & $\begin{array}{c}\text { Baseline } \\
\text { Mean (SD) }\end{array}$ & $\begin{array}{c}\text { Follow-up } \\
\text { Mean (SD) }\end{array}$ & $\begin{array}{c}\text { Change } \\
\text { Mean (SD) }\end{array}$ & P-value \\
\hline Steps per day, number & $4302(2716)$ & $5728(3336)$ & $1426(2687)$ & $<0.0001$ \\
Body mass index, $\mathrm{kg} / \mathrm{m}^{2}$ & $32.1(5.2)$ & $31.8(5.2)$ & $-0.3(1.1)$ & 0.0011 \\
10-foot walk, meters/second & $1.13(0.20)$ & $1.24(0.23)$ & 0.096 & $<0.0001$ \\
Timed Up and Go, seconds & $10.3(2.3)$ & $9.5(2.0)$ & $-0.8(1.2)$ & $<0.0001$ \\
Grip strength, kg & $31.2(9.9)$ & $31.7(9.8)$ & $0.5(4.2)$ & 0.1765 \\
\hline
\end{tabular}

The improvement in gait speed is also clinically significant, and it is expected that these changes will translate, on the long term, into clinically relevant improved outcomes (20). Prior studies have demonstrated the potential usefulness and effectiveness of exercise for the promotion of healthy aging, prevention of frailty $(21-24,9,25)$, and for amelioration of frailty in those who were already frail $(8,26)$. However, the results of the prior studies were mixed which may be partially related to differing follow-up periods as well as methods used for assessing frailty across studies. The Lifestyle Interventions and Independence for Elders (LIFE) study examined the effectiveness of a physical activity intervention in sedentary older adults who were at increased risk for future disability. Initial exploratory findings suggested that the intervention, which included aerobic, strength, flexibility, and balance training, led to improvement in frailty (measured by Fried criteria) (3) over one year follow-up, primarily due to an increase in physical activity in the intervention group (21). Later analysis from this study demonstrated that there was no difference in frailty (measured using an abbreviated frailty criteria) (27) from baseline to follow-up (22). In spite of these conflicting results, the general consensus remains that physical activity is the main intervention for frailty (9).

Our study has some weaknesses, including the fairly small number of patients evaluated, a short follow-up period, and that this was not a randomized controlled trial. We also emphasize that even though individuals improved in frailty on one of the frailty characteristics (walking speed), this may not necessarily reflect a global change in frailty classification (i.e., non-frail, pre-frail, or frail). Further, because the patients referred to the Geriatric Walking Clinic were either self-referred or were referred by their treating PCP, we can only observe the effect of this clinical intervention in motivated individuals, many of them who also have support from their doctor. Further, individuals with medical conditions that would limit safety with exercise were excluded. Therefore, this sample includes relatively healthy older adults. Here, we present the results of a quality improvement program; therefore, a strength of our evaluation is that we have demonstrated the effect of promoting walking for exercise in a "real world" clinical setting. Robust representation of minority participants is a significant strength of our program. Similarly, high rates of diabetes could be seen as a strength as patients with diabetes have higher sedentary behavior.

Prior work has demonstrated that exercise is beneficial for older adults. A meta-analysis which conducted a combined analysis of 1,068 participants in eight clinical trials demonstrated that exercise led to improvement in gait speed, balance, and disability in activities of daily living (8). Current exercise recommendations for older adults include 150 minutes of moderate-intensity aerobic activity (such as brisk walking) each week and muscle strengthening exercises on two or more days per week (28). Practically speaking, however, the majority of older adults are highly sedentary, and these recommendations may be an ambitious goal and difficult to achieve in the clinical setting with other competing needs. In fact, among adults aged 65 to 74 years, only $34 \%$ of men and $17 \%$ of women expend more than 2,000 kcal per week in exercise (29). Physical inactivity and health consequences cost over $11 \%$ of US healthcare expenditures, about $\$ 117$ billion in 2014 alone (30). Further, although the exercise recommendations above are a reasonable starting point, several exercise programs with varied approaches, frequency and duration have led to improvements in muscle strength, gait speed, balance, and falls reduction in older adults (31). Therefore, the optimal exercise program for older adults with varying needs is not always clear and likely depends upon a patient's comorbid medical conditions as well as desired results. Current evidence does support that exercise which is delivered in a prescribed manner concludes in improved results $(11,12)$. Our program adds to the existing literature by showing the feasibility and outcomes of a low impact walking prescription wherein the goals were negotiated with the patient, and ongoing motivational support was provided with weekly phone calls. Improvements in gait speed may have a lasting benefit on individual patients' longevity (32). Best et al., in a recent paper, showed that early change in walking speed was predictive of improvement in chronic physical activity among a group of over 2800 community dwelling older adults, further emphasizing the lasting health effects of the increased gait speed found in this study (33).

In summary, our Geriatric Walking Clinic encourages walking for exercise in a "start low and go slow" manner (a familiar tenet in the practice of geriatric medicine) with frequent monitoring and encouragement. Our findings demonstrate that a low-intensity walking intervention leads 


\section{THE JOURNAL OF FRAILTY \& AGING}

to improvements in gait speed, one of the frailty criteria for slowness even at a short follow-up. This new clinical model may be useful for the promotion of physical activity and for the prevention or amelioration of frailty in older adults.

Funding: Funded by VA T21 Non-Institutional Long Term Care Initiative grant "Activity Promotion Consultation Clinic to Engage High-risk Older Veterans in Regular Walking" (VISN 17-G5984), VA ORH grant "Geriatrics Walking Clinic: Rural Expansion" (VA ORH S2-P00738), and the San Antonio Older Americans Independence Center (NIA/NIH P30 AG044271).

Acknowledgements: Supported by funding from the Veterans Administration (VA) Geriatrics and Extended Care (GEC) T21 Non-Institutional Long Term Care Initiative, the VA Office of Rural Health (ORH), the San Antonio Geriatrics Research, Education and Clinical Center at the South Texas Veterans Health Care System, and the San Antonio Older Americans Independence Center at the University of Texas Health Science Center at San Antonio.

Conflict of Interest: There are no conflicts of interest

Ethical standard: This article details results from a clinical demonstration project and does not contain any study involving human subjects.

Open Access: This article is distributed under the terms of the Creative Commons Attribution 4.0 International License (http://creativecommons.org/licenses/by/4.0/), which permits use, duplication, adaptation, distribution and reproduction in any medium or format, as long as you give appropriate credit to the original author(s) and the source, provide a link to the Creative Commons license and indicate if changes were made.

\section{References}

1. Janssen I, Shepard DS, Katzmarzyk PT, Roubenoff R. The healthcare costs of sarcopenia in the United States. Journal of the American Geriatrics Society. 2004;52(1):80-5

2. Fried LP, Tangen CM, Walston J, Newman AB, Hirsch C, Gottdiener J et al. Frailty in older adults: evidence for a phenotype. The Journals of Gerontology Series A Biological Sciences and Medical Sciences. 2001;56(3):M146-M57.

3. Fried LP, Tangen CM, Walston J, Newman AB, Hirsch C, Gottdiener J et al. Frailty in older adults evidence for a phenotype. The Journals of Gerontology Series A Biological Sciences and Medical Sciences. 2001;56(3):M146-M57.

4. Fugate Woods N, LaCroix AZ, Gray SL, Aragaki A, Cochrane BB, Brunner RL et al. Frailty: emergence and consequences in women aged 65 and older in the Women's Health Initiative Observational Study. Journal of the American Geriatrics Society. 2005;53(8):1321-30.

5. de Rezende LFM, Rey-López JP, Matsudo VKR, do Carmo Luiz O. Sedentary behavior and health outcomes among older adults: a systematic review. BMC public health. 2014;14(1):333

6. Santos DA, Silva AM, Baptista F, Santos R, Vale S, Mota J et al. Sedentary behavior and physical activity are independently related to functional fitness in older adults. Experimental gerontology. 2012;47(12):908-12.

7. Espinoza SE, Hazuda HP. Frailty in Older Mexican-American and EuropeanAmerican Adults: Is There an Ethnic Disparity? Journal of the American Geriatrics Society. 2008;56(9):1744-9.

8. Chou C-H, Hwang C-L, Wu Y-T. Effect of exercise on physical function, daily living activities, and quality of life in the frail older adults: a meta-analysis. Archives of physical medicine and rehabilitation. 2012;93(2):237-44.

9. Liu CK, Fielding RA. Exercise as an intervention for frailty. Clinics in geriatric medicine. 2011;27(1):101-10.

10. Fiatarone MA, O'neill EF, Ryan ND, Clements KM, Solares GR, Nelson ME et al. Exercise training and nutritional supplementation for physical frailty in very elderly people. New England Journal of Medicine. 1994;330(25):1769-75.

11. Petrella RJ, Koval JJ, Cunningham DA, Paterson DH. Can primary care doctors prescribe exercise to improve fitness?: The step test exercise prescription (STEP) project. American journal of preventive medicine. 2003;24(4):316-22.

12. Pinto BM, Goldstein MG, Ashba J, Sciamanna CN, Jette A. Randomized controlled trial of physical activity counseling for older primary care patients. American journal of preventive medicine. 2005;29(4):247-55

13. Padala KPP, P.R.; Taylor, T.; Montgomery, B.; Lensing, S.Y.; Bopp, M.M.; Dubbert, P.M.; Sullivan, D.H. Development and implementation of geriatric walking clinic: a home based individualized Veteran centric program. Federal Practitioner. 2016;33(8):44-7.

14. Resnick B, Ory MG, Hora K, Rogers ME, Page P, Bolin JN et al. A proposal for a new screening paradigm and tool called Exercise Assessment and Screening for You (EASY). Journal of Aging and Physical Activity. 2008;16(2):215-33.

15. Stewart AL, Mills KM, King AC, Haskell WL, Gillis D, Ritter PL. CHAMPS physical activity questionnaire for older adults: outcomes for interventions. Medicine \& Science in Sports \& Exercise. 2001;33(7):1126-41.

16. Podsiadlo D, Richardson S. The timed "Up \& Go": a test of basic functional mobility for frail elderly persons. Journal of the American geriatrics Society. 1991;39(2):142-

17. Royall DR, Cordes JA, Polk M. CLOX: an executive clock drawing task. Journal of Neurology, Neurosurgery \& Psychiatry. 1998;64(5):588-94.

18. Borson S, Scanlan J, Brush M, Vitaliano P, Dokmak A. The mini-cog: a cognitive 'vital signs' measure for dementia screening in multi-lingual elderly. International journal of geriatric psychiatry. 2000;15(11):1021-7.

19. Borkhoff CM, Johnston PR, Stephens D, Atenafu E. The special case of the $2 \times 2$ table: asymptotic unconditional McNemar test can be used to estimate sample size even for analysis based on GEE. J Clin Epidemiol. 2015;68(7):733-9. doi:10.1016/j jclinepi.2014.09.025.

20. Purser JL, Weinberger M, Cohen HJ, Pieper CF, Morey MC, Li T et al. Walking speed predicts health status and hospital costs for frail elderly male veterans. Journal of rehabilitation research \& development. 2005;42(4).

21. Cesari M, Vellas B, Hsu F-C, Newman AB, Doss H, King AC et al. A physical activity intervention to treat the frailty syndrome in older persons-results from the LIFE-P study. The Journals of Gerontology Series A: Biological Sciences and Medical Sciences. 2015;70(2):216-22.

22. Trombetti A, Hars M, Hsu F, et al. Effect of physical activity on frailty: Secondary analysis of a randomized controlled trial. Annals of Internal Medicine. 2018;168(5):309-16. doi:10.7326/M16-2011

23. Villareal DT, Banks M, Sinacore DR, Siener C, Klein S. Effect of weight loss and exercise on frailty in obese older adults. Archives of internal medicine. 2006;166(8):860-6

24. Villareal DT, Chode S, Parimi N, Sinacore DR, Hilton T, Armamento-Villareal R et al. Weight loss, exercise, or both and physical function in obese older adults. N Engl J Med. 2011;364(13):1218-29. doi:10.1056/NEJMoa1008234.

25. Cameron ID, Fairhall N, Langron C, Lockwood K, Monaghan N, Aggar C et al. A multifactorial interdisciplinary intervention reduces frailty in older people: randomized trial. BMC medicine. 2013;11(1):65.

26. Binder EF, Schechtman KB, Ehsani AA, Steger-May K, Brown M, Sinacore DR et al. Effects of exercise training on frailty in community-dwelling older adults: results of a randomized, controlled trial. Journal of the American Geriatrics Society. 2002;50(12):1921-8.

27. Ensrud KE, Ewing SK, Taylor BC, Fink HA, Cawthon PM, Stone KL et al Comparison of 2 frailty indexes for prediction of falls, disability, fractures, and death in older women. Arch Intern Med. 2008;168(4):382-9. doi:10.1001/ archinternmed.2007.113.

28. Prevention CfDCa. Physical activity basics: Physical activity is essential to healthy aging. 2018. https://www.cdc.gov/physicalactivity/basics/older_adults/index.htm. Accessed August 9, 2018

29. Crespo CJ, Keteyian SJ, Heath GW, Sempos CT. Leisure-time physical activity among US adults: results from the Third National Health and Nutrition Examination Survey. Archives of internal medicine. 1996;156(1):93-8.

30. Carlson SA, Fulton JE, Pratt M, Yang Z, Adams EK. Inadequate physical activity and health care expenditures in the United States. Progress in cardiovascular diseases. 2015;57(4):315-23

31. de Labra C, Guimaraes-Pinheiro C, Maseda A, Lorenzo T, Millán-Calenti JC. Effects of physical exercise interventions in frail older adults: a systematic review of randomized controlled trials. BMC geriatrics. 2015;15(1):154.

32. Studenski S, Perera S, Patel K, Rosano C, Faulkner K, Inzitari M et al. Gait speed and survival in older adults. Jama. 2011;305(1):50-8.

33. Best JR, Liu-Ambrose T, Metti AL, Rosso AL, Satterfield S, Studenski S et al Longitudinal associations between walking speed and amount of self-reported time spent walking over a 9-year period in older women and men. The Journals of Gerontology: Series A. 2017;73(9):1265-71. 